\title{
THERMAL NOISE IN SPIN-PHONON SYSTEMS
}

\section{HAROLD A. SABBAGH}

\section{GPO PRICE \\ CFSTI PRICE(S) \$ \\ $\$$}

Hard copy (HC)

Microfiche (MF) _

ff 653 July 65

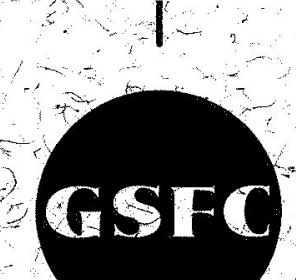

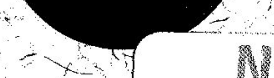

\section{$\$$}

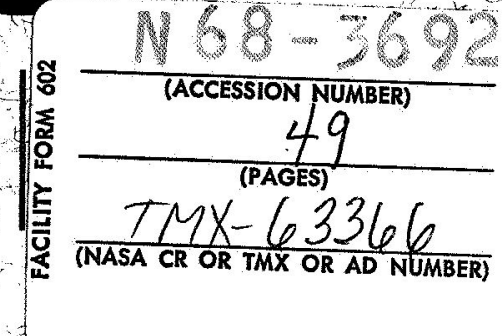

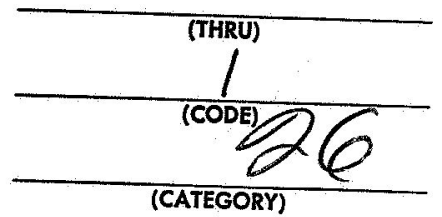

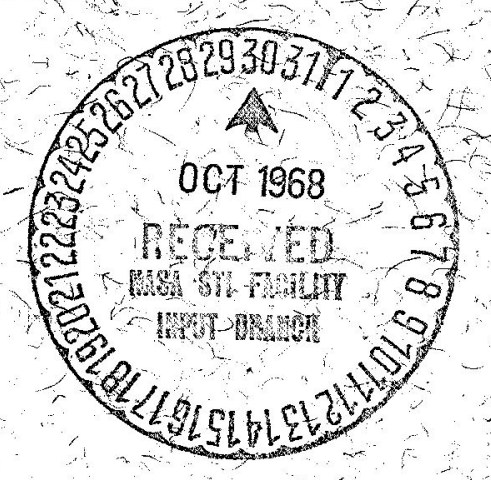


THERMAL NOISE IN SPIN-PHONON SYSTEMS

Harold A. Sabbagh

Rose Polytechnic Institute

September 1968

\author{
GODDARD SPACE FLIGHT CENTER \\ Greenbelt, Maryland
}

The research reported herein was supported in part by NASA Grant: NGR-15-008-004 to Rose Polytechnic Institute. 
THERMAL NOISE IN SPIN-PHONON SYSTEMS

Harold A. Sabbagh

Rose Polytechnic Institute

September 1968

GODDARD SPACE FLIGHT CENTER

Greenbelt, Maryland

The research reported herein was supported in part by NASA Grant: NGR-15-008-004 to Rose Polytechnic Institute. 


\title{
THERMAL NOISE IN SPIN-PHONON SYSTEMS
}

\author{
Harold A. Sabbagh
}

Rose Polytechnic Institute

\begin{abstract}
Starting with the combined lattice and spin-lattice Hamiltonian densities, field equations for transverse elastic waves are derived. An electrical transmission line analog is established which employs an "impedance" function (matrix) calculated using the density-matrix formalism for the interaction of a paramagnetic spin system with acoustic vibrations.

Associated with the impedance function is a continuous distribution of thermal noise "voltage" sources whose statistical characteristics are determined by a form of the (Nyquist) "fluctuation-dissipation" theorem. The significance of positive and negative temperatures on the spectral-density of the noise sources is pointed out.

Application of the theory is made in determining the spatial correlation and spectral densities of the resultant acoustic noise fields in infinite and bounded systems.
\end{abstract}



CONTENTS

$\underline{\text { Page }}$

ABSTRACT ........................... iii

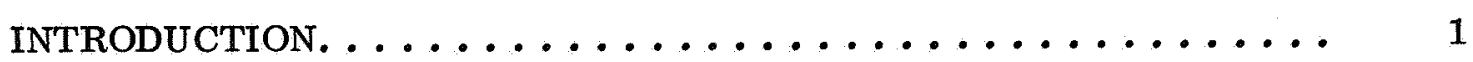

HAMILTONIAN DENSITY FOR A SPIN-PHONON SYSTEM. . . . . . 2

Hamiltonian Density of an Elastic Medium ............. 2

Hamiltonian of a Paramagnetic Spin System (Spin Hamiltonian). . . 3

Interaction Hamiltonian Density (Spin-Lattice Hamiltonian) . . . . 3

CANONICAL FIELD EQUATIONS .................... 6

SUSCEPTIBILITY MATRIX ..................... 8

ELECTRIC TRANSMISSION-LINE ANALOGY . . . . . . . . . . 13

THERMAL NOISE SOURCE ...................... 16

CALCULATION OF OUTPUT NOISE SPECTRAL DENSITIES . . . . . 26

Correlation and Spectral Density of Noise in an Infinite Region. . . 27

Signal Source and Free Termination ............... 34

Signal Source and Matched Termination ............... 39

REFERENCES ............................. 43 


\section{THERMAL NOISE IN SPIN-PHONON SYSTEMS}

\section{INTRODUCTION}

With the advent of acoustical devices in engineering systems, ${ }^{(1)}$ it is desirable to investigate noise properties of phonon systems. A particularly interesting study is that of paramagnetic ions doped into an elastic medium, for this can serve as a model for a spin-phonon maser, adjustable acoustical delayline, etc.

The problem of thermal noise in electromagnetic systems has been investigated (Haus, 1961; Vanwormhaudt and Haus, 1962; Landau and Lifshitz, 1960), and has been applied to electromagnetic masers (Siegman, 1964). It is appropriate to adapt some of the ideas presented in those earlier studies to the problem at hand, namely thermal noise fluctuations in an elastic system.

Our approach to the problem is to determine the lattice and spin-lattice Hamiltonian densities and then derive the canonical field equations appropriate to the model. We then deduce the spin-phonon susceptibility matrix which relates the strain-vector to a spin-induced stress vector and from this determine an electrical transmission-line analogy.

At this point contact can be made with the earlier investigations mentioned above in determining the spectral densities of certain fictitious noise "voltage"

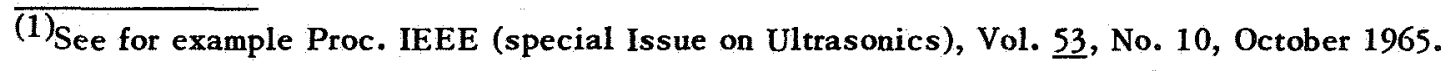


sources. These sources act as inhomogeneous terms in the transmission-line equations, which are solved for either unbounded or bounded configurations using Green's functions. Once the "voltage" and "current" on the transmissionline are determined it is quite straightforward to calculate their spectral-densities.

\section{HAMILTONIAN DENSITY FOR A SPIN-PHONON SYSTEM}

\section{Hamiltonian Density of an Elastic Medium}

The Hamiltonian density of an elastic medium is an expression of energy per unit volume given in terms of momenta, displacements, derivatives of displacements (strains), etc. The total energy (kinetic plus potential) residing within a certain region of space is given by the integral over that region of the Hamiltonian density.

The lattice (elàstic) Hamiltonian density for an isotropic medium is given by (Morse and Feshbach, 1953):

$$
\begin{aligned}
H_{l}=\frac{1}{2 \rho}\left(p_{\mathrm{x}}{ }^{2}\right. & \left.+\mathrm{p}_{\mathrm{y}}{ }^{2}+\mathrm{p}_{\mathrm{z}}{ }^{2}\right)+\frac{\lambda}{2}\left(\frac{\partial \mathrm{u}_{\mathrm{x}}}{\partial \mathrm{x}} \frac{\partial \mathrm{u}_{\mathrm{y}}}{\partial \mathrm{y}}+\frac{\partial \mathrm{u}_{\mathrm{z}}}{\partial \mathrm{z}}\right)^{2} \\
& +\mu\left[\left(\frac{\partial \mathrm{u}_{\mathrm{x}}}{\partial \mathrm{x}}\right)^{2}+\left(\frac{\partial \mathrm{u}_{\mathrm{y}}}{\partial \mathrm{y}}\right)^{2}+\left(\frac{\partial \mathrm{u}_{\mathrm{z}}}{\partial \mathrm{z}}\right)^{2}\right]+\frac{\mu}{2}\left[\left(\frac{\partial \mathrm{u}_{\mathrm{x}}}{\partial \mathrm{y}}+\frac{\partial \mathrm{u}_{\mathrm{y}}}{\partial \mathrm{x}}\right)^{2}\right. \\
& \left.+\left(\frac{\partial \mathrm{u}_{\mathrm{x}}}{\partial \mathrm{z}}+\frac{\partial \mathrm{u}_{\mathrm{z}}}{\partial \mathbf{x}}\right)^{2}+\left(\frac{\partial \mathrm{u}_{\mathrm{y}}}{\partial \mathrm{z}}+\frac{\partial \mathrm{u}_{\mathrm{z}}}{\partial \mathrm{y}}\right)^{2}\right],
\end{aligned}
$$

where $\rho=$ mass density, and $\mu$ and $\lambda$ are the two elastic constants specifying the stress-strain relationship of an elastic medium. $u_{x}, u_{y}, u_{z}$ are the components 
of the vector displacement of the medium at $\mathbf{x}, \mathbf{y}, \mathbf{z}$, while $\mathbf{p}_{\mathbf{x}}=\rho\left(\partial \mathrm{u}_{\mathbf{x}} / \partial \mathrm{t}\right)$, $\mathbf{p}_{\mathbf{y}}=\rho(\partial \mathbf{y} / \partial \mathrm{t}), \mathbf{p}_{\mathbf{z}}=\rho\left(\partial \mathrm{u}_{\mathrm{z}} / \partial \mathrm{t}\right)$ are the components of the momentum density at $\mathrm{x}, \mathrm{y}$ and $\mathrm{z}$.

The momentum terms in (1) are the kinetic energy density while the remaining terms are the potential energy density arising from the stress-strain interaction.

Hamiltonian of a Paramagnetic Spin System (Spin Hamiltonian)

The spin Hamiltonian is a quantum mechanical operator function of spin operators (Bleaney and Stevens, 1953; Bowers and Owen, 1955). Its eigenvalues are the allowed energies of the (unperturbed) paramagnetic ion, and its eigenvectors are the states corresponding to the allowed energies.

The energy levels are important to the determination of operating (resonant) frequencies of paramagnetic devices, such as masers, while the eigenvectors are used to determine the matrix elements, or transition probabilities, which indicate the "strength" of interactions between the elastic system and the spin system.

\section{Interaction Hamiltonian Density (Spin-Lattice Hamiltonian)}

In order to describe the interaction between the elastic and spin systems, one needs a Hamiltonian density which involves elastic variables (displacement, strain, etc.) and spin operators (Tucker, 1965). There are three important conditions that such a Hamiltonian must satisfy (Van Kranendank and Lee, 1966): 
1. Hermiticity: The spin-lattice Hamiltonian density must be Hermitian. This guarantees that the interaction energies (the eigenvalues of the Hamiltonian) will be real. In order that the Hamiltonian be Hermitian it must be a real function of the elastic variables, d.c. magnetic field, and spin operators. All combinations of spin operators appearing in the Hamiltonian must, themselves, be Hermitian.

2. Time-reversal invariance: Because the spin-lattice coupling involves electromagnetic interactions, due to the presence of a spin-vector and an external magnetic field, the spin-lattice Hamiltonian must be invariant to time-reversal. This means that upon changing the signs of the external d.c. magnetic field and spin-vector, the Hamiltonian becomes complex-conjugated.

3. Point symmetry: Finally, the spin-lattice Hamiltonian must be invariant under the group of operations corresponding to the point symmetry of the paramagnetic ion's site in the host crystal. Thus, if we assume that the ion occupies a site of octahedral symmetry within the crystal, the interaction Hamiltonian density must be invariant under the operations of the octahedral group.

These conditions imply that the continuum (long-wavelength) limit there will be four possible forms for the open-lattice Hamiltonian density, of s $f$ :

$$
\mathscr{d}_{\mathrm{s}} \ell_{1}=\mu \mathrm{NA}_{1}\left(\overline{\mathrm{H}}_{0} \cdot \overline{\mathrm{S}}\right) \operatorname{div}(\overline{\mathrm{u}})
$$




$$
\begin{aligned}
& \mathscr{H}_{\mathrm{s} \ell_{2}}=\mu \mathrm{NA}_{2}\left[\left(\overline{\mathrm{H}}_{0} \overline{\mathrm{S}}+\overline{\mathrm{SH}}_{0}\right): \overline{\bar{\epsilon}}-\frac{2}{3}\left(\overline{\mathrm{H}}_{0} \cdot \overline{\mathrm{S}}\right) \operatorname{div}(\overline{\mathrm{u}})\right] \\
& \mathscr{H}_{\mathrm{s} \ell_{3}}=\mu \mathrm{NA}_{3}\left(\overline{\mathrm{H}}_{0} \times \overline{\mathrm{S}}\right) \cdot \operatorname{cur} 1(\overline{\mathrm{u}}) \\
& \mathcal{H}_{\mathrm{s} \ell_{4}}=\mu \mathrm{NA}_{4}\left[\overline{\mathrm{S}} \overline{\mathrm{S}}: \overline{\bar{\epsilon}}-\frac{1}{3} \mathrm{~S}(\mathrm{~S}+1) \operatorname{div}(\overline{\mathrm{u}})\right] .
\end{aligned}
$$

$A_{1}-A_{4}$ are empirically determined constants, $N=$ no. of ions per unit volume, $\bar{H}_{0}$ is the d.c. magnetic field, and $\overline{\mathrm{S}}$ is the ion's spin-vector operator. Also $\epsilon_{\mathrm{ab}}=1 / 2\left(\partial \mathrm{u}_{\mathrm{b}} / \partial \mathrm{x}_{\mathrm{a}}+\partial \mathrm{u}_{\mathrm{a}} / \partial \mathrm{x}_{\mathrm{b}}\right)$ is the strain-tensor and

$$
\overline{\mathbf{S}} \overline{\mathrm{S}}: \overline{\bar{\epsilon}} \cdot=\sum_{\mathrm{a}, \mathrm{b}} \mathrm{S}_{\mathrm{a}} \mathrm{S}_{\mathrm{b}} \epsilon_{\mathrm{b} a} .
$$

The first three forms in (2) are linear in each of the variables $\overline{\mathrm{H}}_{0}$ and $\overline{\mathrm{S}}$, whereas the fourth is independent of $\overline{\mathrm{H}}_{0}$ but depends quadratically on $\overline{\mathrm{S}}$. Contrast these possibilities with the single Hamiltonian, $g \overline{\beta \mathrm{H}} \cdot \overline{\mathrm{S}}$, of electromagneticspin interactions.

The total Hamiltonian density for the combined spin-elastic system is given by the sum of the three Hamiltonians mentioned so far:

$$
H_{t}=f_{1}+f_{s}+f_{s} \ell \text {, }
$$

where $f_{s}$ is the spin-Hamiltonian.

As (3) stands it is a mixture of a classical Hamiltonian, $\mathscr{H}_{1}$, and quantummechanical Hamiltonians, $\mathcal{f}_{\mathrm{s}}$ and $\mathcal{f}_{\mathrm{s}} \ell$, involving spin operators. Eventually 
we will take quantum average values of these operators and proceed classically.

\section{CANONICAL FIELD EQUATIONS}

One important reason for expressing the mathematical model of a physical system in terms of a Hamiltonian density is that one may derive the differential equations for a variety of different subsystems of the given system in a canonical form.

Because the spin subsystem in our problem requires a quantum-mechanical Hamiltonian formulation, it is natural to develop the elastic subsystem in a Hamiltonian manner, also. As previously mentioned, the latter Hamiltonian is classical, i.e., it does not involve non-commuting operators.

From here on we consider transverse vibrations propagating in the $z$-direction, the assumed direction of the d.c. field $H_{0}$. Thus, in (1) and (3) we set $\mathrm{p}_{\mathrm{z}}, \partial / \partial \mathrm{x}, \partial / \partial \mathrm{y}, \mathrm{u}_{\mathrm{z}}, \mathrm{H}_{0_{\mathrm{x}}}$ and $\mathrm{H}_{\mathrm{y}_{\mathrm{y}}}$ equal to zero. Note also that terms involving $\operatorname{div}(\bar{u})$ vanish. The resultant combination, $H^{\prime}$, of the lattice plus spinlattice Hamiltonians becomes, after taking quantum-average values

$$
\begin{aligned}
H^{\prime}= & \frac{1}{2 \rho}\left(\mathbf{p}_{\mathbf{x}}{ }^{2}+\mathbf{p}_{\mathbf{y}}{ }^{2}\right)+\frac{\mu}{2}\left[\left(\frac{\partial \mathrm{u}_{\mathbf{x}}}{\partial \mathrm{z}}\right)^{2}\left(\frac{\partial \mathrm{u}_{\mathbf{y}}}{\partial \mathrm{z}}\right)^{2}\right] \\
& +\mathrm{M}_{\mathrm{x}} \frac{\partial \mathrm{u}_{\mathbf{x}}}{\partial \mathrm{z}}+\mathrm{M}_{\mathrm{y}} \frac{\partial \mathrm{u}_{\mathbf{y}}}{\partial \mathrm{z}},
\end{aligned}
$$


where

$$
\begin{aligned}
& M_{x}=\left\langle\left(A_{2}+A_{3}\right) H_{0} S_{x}+A_{4}\left(S_{z} S_{x}+S_{x} S_{z}\right)\right\rangle \mu^{N} \\
& M_{y}=\left\langle\left(A_{2}+A_{3}\right) H_{0} S_{y}+A_{4}\left(S_{z} S_{y}+S_{y} S_{z}\right)\right\rangle \mu N,
\end{aligned}
$$

and the brackets denote quantum-averaging.

Hamilton's canonical equations are [Morse and Feshbach, 1953].

$\dot{\mathrm{u}}_{\mathrm{x}}=\frac{\partial \mathrm{H}^{\prime}}{\partial \mathrm{p}_{\mathrm{x}}} ; \quad \dot{\mathrm{p}}_{\mathrm{x}}=\frac{\partial}{\partial \mathrm{x}} \frac{\partial \mathrm{H}^{\prime}}{\partial\left(\frac{\partial \mathrm{u}_{\mathrm{x}}}{\partial \mathrm{x}}\right)}+\frac{\partial}{\partial \mathrm{y}} \frac{\partial \mathrm{H}^{\prime}}{\partial\left(\frac{\partial \mathrm{u}_{\mathrm{x}}}{\partial \mathrm{y}}\right)}+\frac{\partial}{\partial \mathrm{z}} \frac{\partial \mathrm{H}^{\prime}}{\partial\left(\frac{\partial \mathrm{u}_{\mathrm{x}}}{\partial \mathrm{z}}\right)}-\frac{\partial \mathrm{H}^{\prime}}{\partial \mathrm{u}_{\mathrm{x}}}$

$\dot{\mathrm{u}}_{\mathrm{y}}=\frac{\partial \mathrm{H}^{\prime}}{\partial \mathrm{p}_{\mathrm{y}}} ; \quad \dot{\mathrm{p}}_{\mathrm{y}}=\frac{\partial}{\partial \mathrm{x}} \frac{\partial \mathrm{H}^{\prime}}{\partial\left(\frac{\partial \mathrm{u}_{\mathrm{y}}}{\partial \mathbf{x}}\right)}+\frac{\partial}{\partial \mathrm{y}} \frac{\partial \mathrm{H}^{\prime}}{\partial\left(\frac{\partial \mathrm{u}_{\mathrm{y}}}{\partial \mathrm{y}}\right)}+\frac{\partial}{\partial \mathrm{z}} \frac{\partial \mathrm{H}^{\prime}}{\partial\left(\frac{\partial \mathrm{u}_{\mathrm{y}}}{\partial z}\right)}-\frac{\partial \mathrm{H}^{\prime}}{\partial \mathrm{u}_{\mathrm{y}}}$

$\dot{\mathrm{u}}_{\mathrm{z}}=\frac{\partial \mathrm{H}^{\prime}}{\partial \mathrm{p}_{\mathrm{z}}} ; \quad \dot{\mathrm{p}}_{\mathrm{z}}=\frac{\partial}{\partial \mathrm{x}} \frac{\partial \mathrm{H}^{\prime}}{\partial\left(\frac{\partial \mathrm{u}_{\mathrm{z}}}{\partial \mathrm{x}}\right)}+\frac{\partial}{\partial \mathrm{y}} \frac{\partial \mathrm{H}^{\prime}}{\partial\left(\frac{\partial \mathrm{u}_{\mathrm{z}}}{\partial \mathrm{y}}\right)}+\frac{\partial}{\partial \mathrm{z}} \frac{\partial \mathrm{H}^{\prime}}{\partial\left(\frac{\partial \mathrm{u}_{\mathrm{z}}}{\partial \mathrm{z}}\right)}-\frac{\partial \mathrm{H}^{\prime}}{\partial \mathrm{u}_{z}}$

where a dot denotes time-differentiation.

Upon substituting (4) into (6), the following system is obtained

$$
\begin{aligned}
& \dot{\mathrm{u}}_{\mathrm{x}}=\mathrm{p}_{\mathrm{x}} / \rho ; \quad \dot{\mathrm{p}}_{\mathrm{x}}=\mu \frac{\partial^{2} \mathrm{u}_{\mathrm{x}}}{\partial \mathrm{z}^{2}}+\frac{\partial \mathrm{M}_{\mathbf{x}}}{\partial \mathrm{z}} \\
& \dot{\mathrm{u}}_{\mathbf{y}}=\mathrm{p}_{\mathrm{y}} / \rho ; \quad \dot{\mathrm{p}}_{\mathbf{y}}=\mu \frac{\partial^{2} \mathrm{u}_{\mathbf{y}}}{\partial \mathrm{z}^{2}}+\frac{\partial \mathrm{M}_{\mathbf{y}}}{\partial \mathrm{z}} .
\end{aligned}
$$


All terms in (7) are classical; quantum-mechanical spin operators have been replaced by their average values. Equation (7) shows that the source of elastic vibrations is the gradient of the paramagnetic stress functions $M_{x}$ and $M_{y}$. This agrees with the statements of Jacobsen (1960) and Seavey (1965) regarding piezoelectric and magnetic production of acoustical vibrations, respectively.

$M_{x}$ and $M_{y}$ are not given a priori. They depend on $\partial u_{x} / \partial z$ and $\partial u_{y} / \partial z$ (actu: the randomly fluctuating parts of $M_{x}$ and $M_{y}$ can be considered to be given functions, independent of $\partial u_{x} / \partial z$ and $\partial u_{y} / \partial z$. We show this in another section). After determining $M_{x}$ and $M_{y}$ as functions of $\partial u_{x} / \partial z$ and $\partial u_{y} / \partial z$, we substitute the results back into (7) to obtain wave equations for $u_{x}$ and $u_{y}$. The relationship between $M_{x}, M_{y}$ and $\partial u_{x} / \partial z, \partial u_{y} / \partial z$ involves the spin-phonon susceptibilit matrix.

\section{SUSCEPTIBILITY MATRIX}

Because the spin system is an atomic system we must resort to quantum mechanical principles for calculating average values of the pertinent spin operators. A useful tool for this purpose is the density matrix (Sabbagh, 196 Slichter, 1963).

Every quantum-mechanical operator may be given a matrix representat: by choosing a complete, ortho-normal basis system of vectors. The basis th we will use will be the eigenvectors of the spin-Hamiltonian. 
The average value of operator, $A$, then is given by

$$
\langle A\rangle=\operatorname{Tr}\left[\rho_{\mathrm{ij}}\right]\left[\mathrm{A}_{\mathrm{jn}}\right],
$$

where $\operatorname{Tr}$ denotes the trace (sum of diagonal elements) of a matrix, $\rho_{i j}$ is the

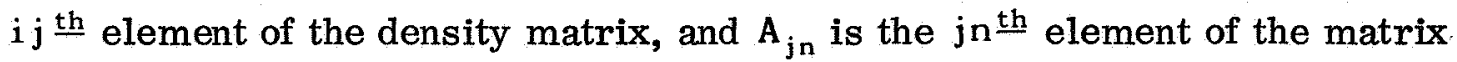
representation of the operator A. If there are $\mathrm{p}$ energy levels in the paramagnetic spin system, all matrices will be $\mathrm{P} \times \mathrm{P}$.

To a first (linear) approximation the diagonal elements, $\rho_{\mathrm{mm}}, \mathrm{m}=1 \ldots, \mathrm{p}$ of the density matrix remain constant at their thermal-equilibrium values, $\rho_{\mathrm{mm}}^{(\mathrm{T})}$. The off-diagonal elements, to the same order of approximation, satisfy the system of linear equations

$$
\frac{\mathrm{d} \rho_{\mathrm{mn}}}{\mathrm{dt}}=\left(\mathbf{j} \omega_{\mathrm{mn}}-\frac{1}{\tau_{\mathrm{mn}}}\right) \rho_{\mathrm{mn}}+\frac{\mathbf{j}}{\hbar}\left(\rho_{\mathrm{mm}}^{(\mathrm{T})}-\rho_{\mathrm{nn}}^{(\mathrm{T})}\right) H_{\mathrm{s}} l_{\mathrm{mn}},
$$

where $\mathrm{m} \neq \mathrm{n}$ and $\mathrm{m}, \mathrm{n}=1, \ldots, \mathrm{p} . \omega_{\mathrm{mn}}=\mathrm{E}_{\mathrm{n}}-\mathrm{E}_{\mathrm{m}} / \mathrm{h}$ is the frequency corresponding to the difference between the $n \frac{\text { th }}{}$ and $m \frac{\text { th }}{}$ unperturbed energy levels. The $\tau^{\prime} \mathbf{s}$ are time-constants related to the return of the spin system to thermal equilibrium after a transient perturbation. Finally, $\not_{\mathrm{s}} \ell_{\mathrm{mn}}$ is the $\mathrm{mn}$ th matrix element of the spin-lattice Hamiltonian operator. All matrix elements are between the unperturbed eigenstates of the spin-Hamiltonian. 
In the sinusoidal steady-state $f_{\mathrm{s}} \ell_{\mathrm{mn}}$, for a single ion, becomes (see (5))

$$
\begin{aligned}
H_{s} \ell_{m n}(t)= & \left(S_{x_{m n}^{\prime}}^{\prime} \mu \frac{\partial U_{x}}{\partial z}+S_{y_{m n}^{\prime}}^{\prime} \mu \frac{\partial U_{y}}{\partial z}\right) e^{h \omega t} \\
& +\left(S_{x_{m n}}^{\prime} \mu \frac{\partial U_{x}^{*}}{\partial z}+S_{y_{m n}^{\prime}}^{\prime} \mu \frac{\partial U_{y}^{*}}{\partial z}\right) e^{-j \omega t}
\end{aligned}
$$

The asterisk denotes complex conjugate and the field amplitudes $U_{x}, U_{y}$ are independent of time. In (10) we have made the definitions

$$
\begin{aligned}
& S_{x}^{\prime}=\left(A_{2}+A_{3}\right) H_{0} S_{x}+A_{4}\left(S_{z} S_{x}+S_{x} S_{z}\right) \\
& S_{y}^{\prime}=\left(A_{2}+A_{3}\right) H_{0} S_{y}+A_{4}\left(S_{z} S_{y}+S_{y} S_{z}\right) .
\end{aligned}
$$

The solution of (9), given (10), is easily obtained because of the sinusoidal, steady-state condition (it is equally easily obtained as a convolution integral, as well):

$\rho_{m n}(t)=\frac{j\left(\rho_{m m}^{(T)}-\rho_{n n}^{(T)}\right) \mu}{\bar{h}}\left[\frac{\left(S_{x_{\ell m}^{\prime}}^{\prime} \frac{\partial U_{x}}{\partial z}+S_{y_{\ell m}^{\prime}}^{\prime} \frac{\partial U_{y}}{\partial z}\right)}{D^{-}} e^{j \omega t}\right.$

$$
\left.+\frac{\left(S_{x_{\ell m}^{\prime}}^{\prime} \frac{\partial U_{x}^{*}}{\partial z}+S_{y_{\ell m}^{\prime}}^{\prime} \frac{\partial U_{y}^{*}}{\partial z}\right)}{D^{+}} e^{-j \omega t}\right]
$$


where

$$
\begin{aligned}
& D^{-}=-j\left(\omega \ell_{m}-\omega\right)+\frac{1}{\tau \ell_{m}} \\
& D^{+}=-j\left(\omega \ell_{m}+\omega\right)+\frac{1}{\tau \ell_{m}} .
\end{aligned}
$$

Comparing (5) and (8) we see that

$$
\begin{aligned}
& \mathbf{M}_{\mathbf{x}}=\mu \mathrm{N} \operatorname{Tr}\left[\rho_{\mathbf{i} \mathbf{j}}\right]\left[\mathbf{S}_{\mathbf{x} \ell_{\mathrm{m}}}^{\prime}\right] \\
& \mathbf{M}_{\mathbf{y}}=\mu \mathbf{N} \operatorname{Tr}\left[\rho_{\mathbf{i} \mathbf{j}}\right]\left[\mathbf{S}_{\mathbf{y}_{\ell_{\mathbf{m}}}^{\prime}}\right] .
\end{aligned}
$$

After carrying out the operations implied in (14) (using (12), of course), dropping any time-independent terms and rearranging, we get

$$
\begin{aligned}
& M_{x}=a_{x x} \mu \frac{\partial U_{x}}{\partial z} e^{j \omega t}+a_{x y} \mu \frac{\partial U_{y}}{\partial z} e^{j \omega t}+\text { complex conjugate } \\
& M_{y}=a_{y x} \mu \frac{\partial U_{x}}{\partial z} e^{j \omega t}+a_{y y} \mu \frac{\partial U_{y}}{\partial z} e^{j \omega t}+\text { complete conjugate } .
\end{aligned}
$$

In (15)

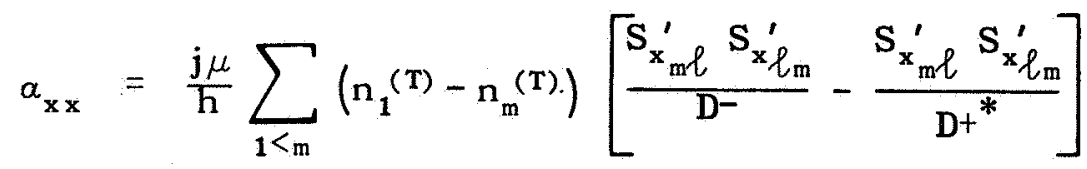

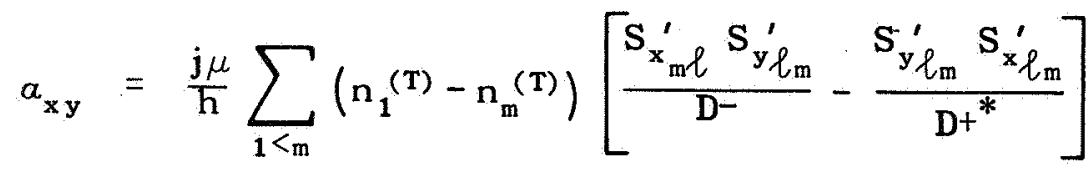




$$
\begin{aligned}
& a_{y x}=\frac{j \mu}{h} \sum_{1<m}\left(n_{1}{ }^{(T)}-n_{m}{ }^{(T)}\right)\left[\frac{S_{y_{m} \ell}^{\prime} S_{x_{\ell m}^{\prime}}^{\prime}}{D^{-}}-\frac{S_{x_{m} \ell}^{\prime} S_{y^{\prime} \ell_{m}}^{\prime}}{D+^{*}}\right] \\
& \alpha_{y y}=\frac{j \mu}{h} \sum_{i<m}\left(n_{1}(T)-n_{m}{ }^{(T)}\right)\left[\frac{S_{y_{m} \ell}^{\prime} S_{y_{\ell m}^{\prime}}^{\prime}}{D^{-}}-\frac{S_{y_{m} \ell}^{\prime} S_{y^{\prime} \ell_{m}}^{\prime}}{D^{+}{ }^{*}}\right]
\end{aligned}
$$

$n_{1}{ }^{(T)}=$ thermal equilibrium no. density of ions in state 1 , etc.

The two-by-two matrix with entries $\alpha_{x x}, a_{x y}, a_{y x}$ and $a_{y y}$ is the spin-phonon susceptibility matrix.

Consider $\alpha_{x x}$, for it is typical of the other three functions. It consists of a summation over pairs of energy levels (with $1<\mathrm{m}$ ) of terms whose real and imaginary parts are of the form

$$
\begin{aligned}
& \text { Real part }=\frac{-2 \mu}{\bar{\hbar}}\left(n_{1}{ }^{(T)}-n_{m}{ }_{m}^{(T)}\right)\left|S \ell_{m}^{\prime}\right|^{2}\left[\frac{\omega_{\ell_{m}}\left(\omega_{\ell m}^{2}-\omega^{2}\right)}{\left(\omega_{\ell_{m}}^{2}-\omega^{2}\right)^{2}+\frac{4 \omega^{2}}{\tau l_{m}^{2}}}\right] \\
& \text { Imaginary part }=\frac{2 \mu}{\hbar}\left(\mathrm{n}_{1}{ }_{1}^{(\mathrm{T})}-\mathrm{n}_{\mathrm{m}}{ }^{(\mathrm{T})}\right)\left|\mathrm{S}_{\ell_{\mathrm{m}}^{\prime}}\right|^{2}\left[\frac{2 \omega \ell_{\mathrm{m}} \omega / \tau \ell_{\mathrm{m}}}{\left(\omega_{\ell_{\mathrm{m}}}^{2}-\omega^{2}\right)^{2}+\frac{4 \omega^{2}}{\tau_{\ell_{\mathrm{m}}^{2}}^{2}}}\right]
\end{aligned}
$$

We see quite clearly the role played by the matrix elements $S_{\ell_{m}^{\prime}}^{\prime}$ in determining the interaction strength between spins and phonons.

The functions shown in (17) are reminiscent of the real and imaginary parts of immitance functions of RLC circuits. $\omega^{\omega} \ell_{\mathrm{m}}$ is the resonant frequency of the resonant circuit corresponding to the $\ell_{m}^{\text {th }}$ pair of energy levels and $2 / \tau \ell_{m}$ is the 
half-power bandwidth of this same "circuit." We conclude, therefore, that the spin-phonon susceptibility functions are analogous to a series combination of RLC resonant circuits.

\section{ELECTRIC TRANSMISSION-LINE ANALOGY}

To develop the electric transmission-line analogy we start with (7), which may be combined to give

$$
\begin{aligned}
\rho \frac{\partial^{2} \mathrm{u}_{\mathrm{x}}}{\partial \mathrm{t}^{2}} & =\mu \frac{\partial^{2} \mathrm{u}_{\mathrm{x}}}{\partial \mathrm{z}^{2}}+\frac{\partial \mathrm{M}_{\mathrm{x}}}{\partial \mathrm{z}} \\
\rho \frac{\partial^{2} \mathrm{u}_{\mathrm{y}}}{\partial \mathrm{t}^{2}} & =\mu \frac{\partial^{2} \mathrm{u}_{\mathrm{y}}}{\partial \mathrm{z}^{2}}+\frac{\partial \mathrm{M}_{\mathrm{y}}}{\partial \mathrm{z}}
\end{aligned}
$$

We establish the analogies

$$
\begin{array}{ll}
\frac{\partial \mathbf{u}_{\mathbf{x}}}{\partial \mathrm{t}} \sim \mathrm{i}_{\mathbf{x}} ; & \mu \frac{\partial \mathrm{u}_{\mathbf{x}}}{\partial \mathrm{z}} \sim \mathrm{v}_{\mathbf{x}} \\
\frac{\partial \mathrm{u}_{\mathrm{y}}}{\partial \mathrm{t}} \sim \mathrm{i}_{\mathbf{y}} ; & \mu \frac{\partial \mathrm{u}_{\mathbf{y}}}{\partial \mathrm{z}} \sim \mathrm{v}_{\mathbf{y}} .
\end{array}
$$

Note that when $\mu \partial \mathrm{u} / \partial \mathrm{z}$ (= stress) is multiplied by $\partial \mathrm{u} / \partial \mathrm{t}$ (velocity), power/ area is obtained. If we imagine one-dimensional wave propagation through a unit cross-section, then stress $\times$ velocity $\times \mathrm{lm}^{2}$ yields power which is $\mathrm{v} \times \mathrm{i}$. Hence, the aptriess of the analogy. 
In terms of the "electrical" variables, (18) becomes

$$
\begin{aligned}
& \rho \frac{\partial \mathbf{i}_{\mathbf{x}}}{\partial \mathrm{t}}=\frac{\partial v_{\mathbf{x}}}{\partial \mathbf{z}}+\frac{\partial \mathrm{M}_{\mathbf{x}}}{\partial \mathbf{z}} ; \quad \frac{1}{\mu} \frac{\partial v_{\mathbf{x}}}{\partial \mathrm{t}}=\frac{\partial \mathbf{i}_{\mathbf{x}}}{\partial \mathbf{z}} \\
& \rho \frac{\partial \dot{i}_{y}}{\partial t}=\frac{\partial v_{y}}{\partial z}+\frac{\partial M_{y}}{\partial z} ; \quad \frac{1}{\mu} \frac{\partial v_{y}}{\partial t}=\frac{\partial \dot{i}_{y}}{\partial t} .
\end{aligned}
$$

Upon taking the Fourier transform of (20) and using (15) we get the vectormatrix equations

$$
\begin{aligned}
& j \omega \rho\left[\begin{array}{c}
I_{x} \\
I_{y}
\end{array}\right]=\left[\begin{array}{cc}
1+a_{x x} & a_{x y} \\
a_{y x} & 1+a_{y y}
\end{array}\right]\left[\begin{array}{l}
d_{x} / d_{z} \\
d_{y} / d_{z}
\end{array}\right] \\
& j \omega \mu\left[\begin{array}{l}
V_{x} \\
V_{y}
\end{array}\right]=\left[\begin{array}{l}
d I_{x} / d_{z} \\
d I_{y} / d_{z}
\end{array}\right] .
\end{aligned}
$$

To simplify matters, we assume a wave linearity polarized in the $\mathrm{x}$-direction. Therefore, after setting $v_{y}=I_{y}=0$ and dropping subscripts, (21) becomes

$$
\begin{aligned}
j \frac{\omega \rho}{1+a} I & =\frac{d V}{d_{z}} \\
j \frac{\omega}{\mu} V & =\frac{D I}{d_{z}} .
\end{aligned}
$$


These equations are obtained from the incremental transmission-line configuration shown in Figure 1(a). Observe the series impedance

$$
\frac{j \omega \rho \Delta z}{1+a}=\frac{j \omega \rho \Delta z\left(1+a_{R-j} a_{I}\right)}{|1+a|^{2}},
$$

where $a_{R}$ is the real-part and $a_{I}$ the imaginary-part of $\alpha$, and shunt admittance j $\omega / \mu \Delta$.

When we recall the summations appearing in (16), we may redraw Figure 1(a) using a series combination of impedances as in Figure 1(b). Each impedance is associated with an energy-level pair as in (17).

Now that we have established an electrical analogue, it is rather straightforward to develop the thermal noise source associated with the dissipative mechanism of the transmission-line.

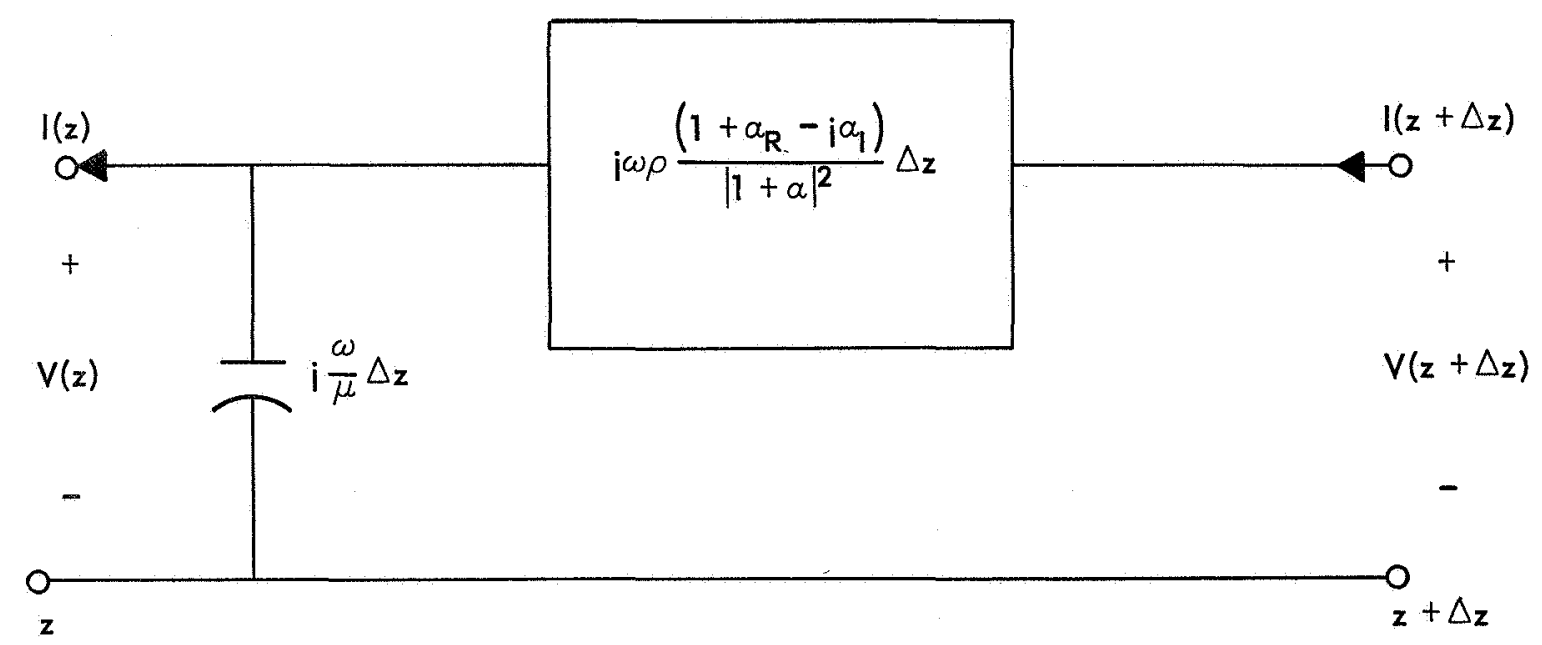

Figure 1(a). Incremental Section of Analogous Tran'smissionline Yielding Equations (22) 


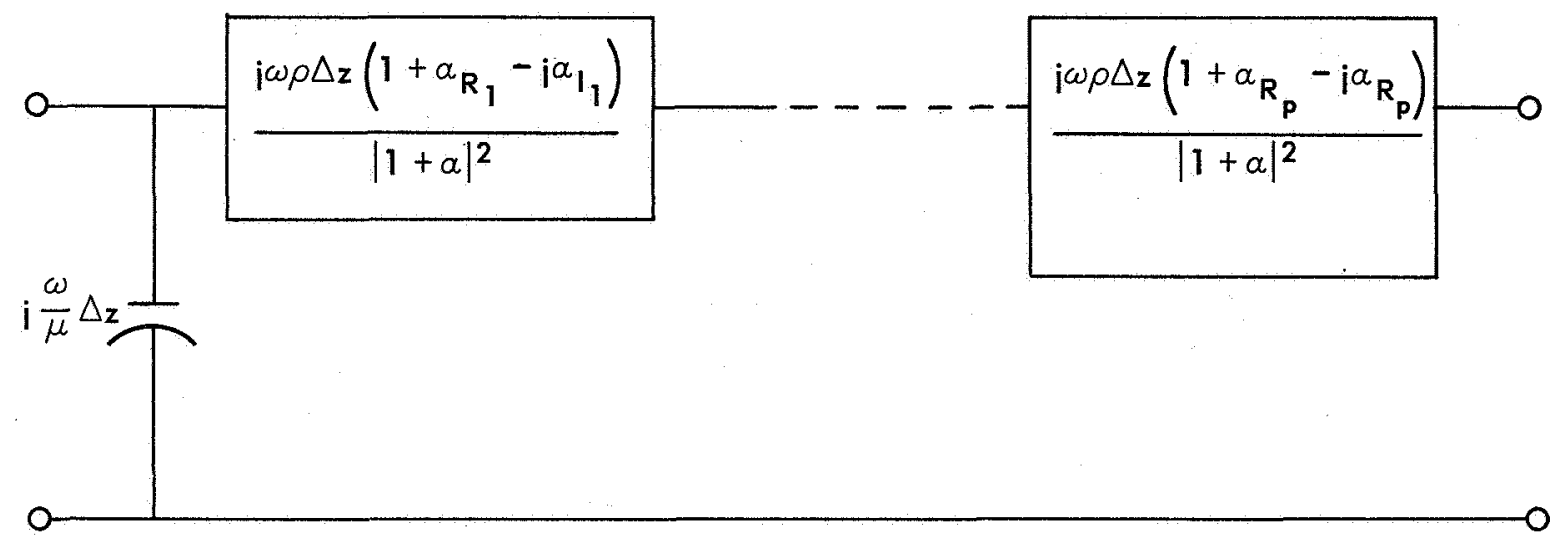

Figure 1(b). Illustrating Expansion of Impedance into a Series Combination in Accordance With (16). Each Block Corresponds to an Energy-level Pair.

\section{THERMAL NOISE SOURCE}

An expression for power-dissipation in terms of a may be obtained from (22) by multiplying the top equation by $\mathrm{I}^{*}$, conjugating the bottom, multiplying the result by $\mathrm{V}$, then adding the two equations and taking one-half of the real-part:

$$
\frac{\mathrm{dP}}{\mathrm{dz}}=\frac{1}{2} \frac{\omega \rho a_{\mathrm{I}}}{|1+\alpha|^{2}}|\mathrm{I}|^{2}=\frac{|\mathrm{I}|^{2}}{2|1+\alpha|^{2}} \omega \rho \sum_{\mathrm{i}=1}^{\mathrm{P}} a_{\mathrm{I}_{\mathrm{i}}} .
$$

The second equality in (23) shows that each energy-level pair contributes to the overall change in power along the line. If $\alpha_{I_{i}}$ is negative for the $i$ th energy-level pair, then that pair contributes gain (maser action at the corresponding resonant frequency). Otherwise we get dissipation"(or absorption) of power.

Now we shall show that $a_{I_{i}}$ is associated with the spectral density of noise sources arising from the series impedances. The generalized Nyquist theorem (Davenport and Root, 1958; Twiss, 1955) asserts that the thermal noise associated 
with a lossy network at temperature $T$ is equivalent to fictitious noise sources connected to the terminals of the network. The spectral densities of the sources are related to the dissipative properties of the network. This result, general as it is for electrical networks, is a special case of the "fluctuation-dissipation" theorem satisfied by all physical systems in thermal equilibrium (Landau and Lifshitz, 1958; de Groot and Mazur, 1962).

By the spectral density of a random process, $x(t)$, we mean

$$
S_{x}(\omega)=\lim _{T \rightarrow 00} E\left[\left|X_{T}(\omega)\right|^{2}\right]
$$

where

$$
X_{T}(\omega)=\frac{1}{\sqrt{2 T}} \int_{-T}^{T} x(t) e^{-j \omega t} d t
$$

and $\mathrm{E}[\mathrm{]}$ implies the expected value of whatever is within the braces. Throughout this report when we speak of the Fourier transform of a function we mean (25).

The significance of the spectral density lies in the fact that the expected mean-square value of $x(t)$ is given by

$$
E\left[\overline{x^{2}(t)}\right]=\frac{1}{2 \pi} \int_{-\infty}^{\infty} S_{x}(\omega) d \omega
$$

By the "one-sided" spectral density we mean $2 S_{x}(\omega)$ with the integration in (26) extending only over positive frequencies. 
Before determining the noise sources associated with each block in Figure 1(b) we make the following preparatory remarks. The noise properties depend on the "temperature" of each block. If the spin system were in thermal equilibrium with the lattice, each energy level would be populated according to the Boltzmann distribution with a single temperature $\mathrm{T}$. Then the ratio of the populations of any pair of energy levels would be simply

$$
\frac{n_{j}}{n_{i}}=\exp \frac{E_{i}-E_{j}}{k T}
$$

where $k$ is Boltzmann's constant and $E_{i}$ and $E_{j}$ are the energies of the $i \stackrel{\text { th }}{\text { and }}$ $\mathrm{j}$ th levels.

In the presence of an external source (such as a pump in a phonon maser), however, the distribution of populations will be altered radically. For example, one pair of energy levels may have an inverted population (more ions in the higher than lower energy level), and another may have equal populations etc.

If we assume that we may always define an equilibrium temperature between pairs of energy levels so that (27) is satisfied, then there will be as many equilibrium temperatures associated with the spin system as there are pairs of energy levels. In the examples above the inverted populations correspond to a negative temperature, while the equally populated pair of energy levels corresponds to infinite temperature.

An important result is that there is a correspondence between $\alpha_{I_{i}}$ and $T_{i}$,

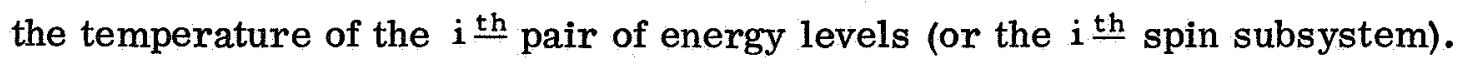


When $\mathrm{T}_{i}>0,(17)$ and (27) show that $\alpha_{I_{i}}>0 ; T_{i}>0$ corresponds to $\alpha_{I_{i}}<0$, and, finally, when $T_{i}=\infty$, then $\alpha_{I_{i}}=0$. These results hold for positive frequencies. Because $\alpha_{I_{i}}$ is an odd function of $\omega$, the reverse of each holds for negative frequencies. From (23), however, we note that the significant function is $\omega \alpha_{I_{i}}$, which is positive, negative, or zero, corresponding to the above temperature ranges, at all frequencies.

In order to determine the spectral densities of the fictitious noise sources associated with each impedance block, we remove the $\mathrm{m}$ th box in Figure 1(b) and connect a series noise voltage source to account for the thermal noise fluctuations at temperature $\mathrm{T}_{\mathrm{m}}$ (see Figure 2). To this combination we connect a resistor $R_{m} \Delta z$ at temperature $T_{m}$ (Figure 3(a)).

The spectral density of noise power delivered to $R_{m} \Delta z$ is easily calculated to be

$$
S_{R}=\frac{1}{2} \frac{S_{m}(\omega) R_{m} \Delta z}{\left(R_{m}+Z_{m}\right)\left(R_{m}+Z_{m}^{*}\right)}
$$

where $S_{m}(\omega)$ is the spectral density of $e_{m}$.

The resistor at temperature $T_{m}$ is, itself, a noise source and may be considered to be a voltage source $e_{R} \Delta z$ in series with $R_{m} \Delta z$ (see Figure $3(b)$ ). The spectral density of the noise power delivered by the resistor to $Z_{M} \Delta z$ is

$$
S_{Z}=\frac{1}{2} \frac{S_{e_{R}}(\omega) \operatorname{Re}\left(Z_{m}\right) \Delta z}{\left(R_{m}+Z_{m}\right)\left(R_{m}+Z_{m}^{*}\right)}
$$


At thermal equilibrium (28) and (29) must be equal, which means that

$$
S_{m}(\omega)=\frac{S_{e_{R}}(\omega)}{R_{m}} \cdot \frac{\omega \rho \alpha I_{m}}{|1+\alpha|^{2}} .
$$

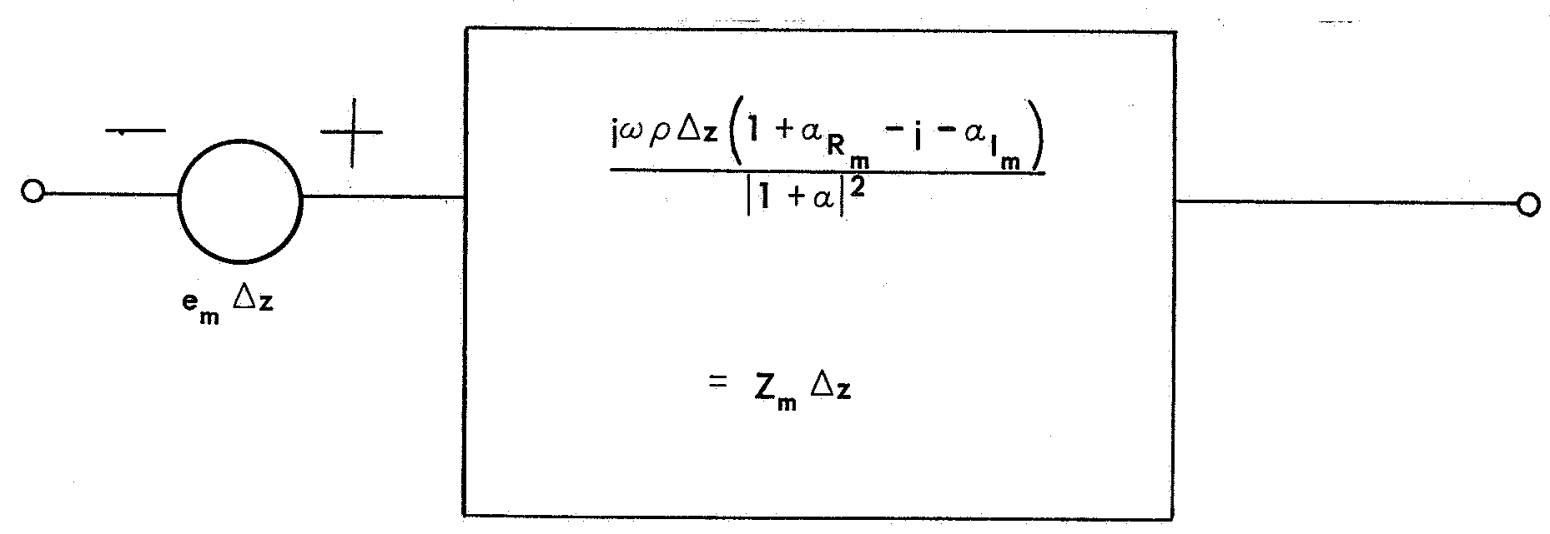

Figure 2. $m^{\text {th }}$ Noise Voltage Source in Series with $m$ th Impedance Block at Temperature $T_{m}$.

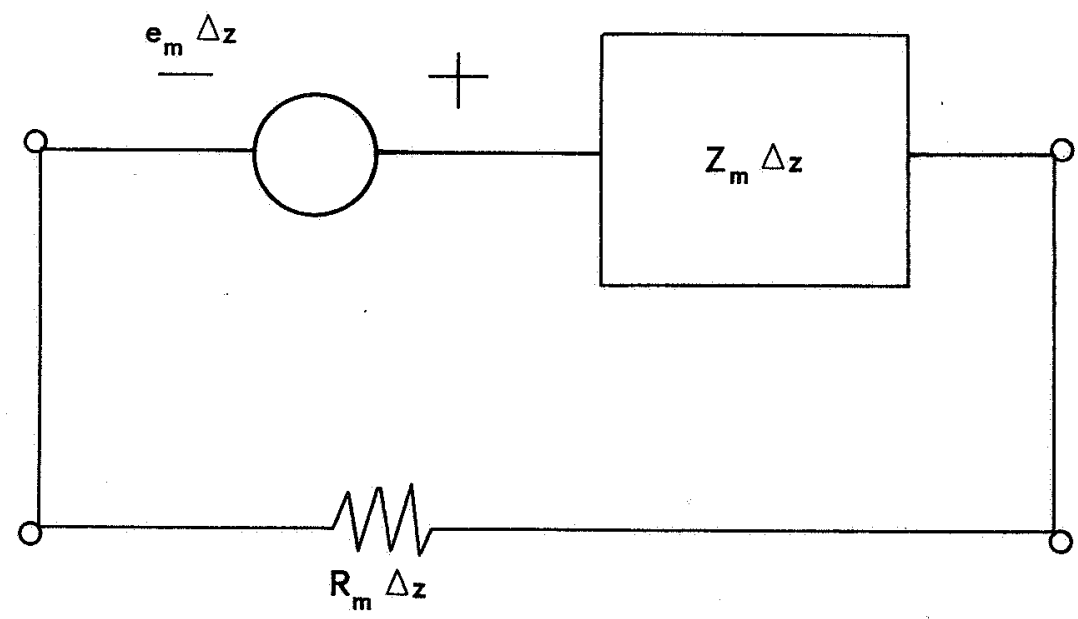

Figure 3(a). Circuit for Calculating Noise Power Delivered to $R_{m} \Delta z$ at Temperature $T_{m^{*}}$. 


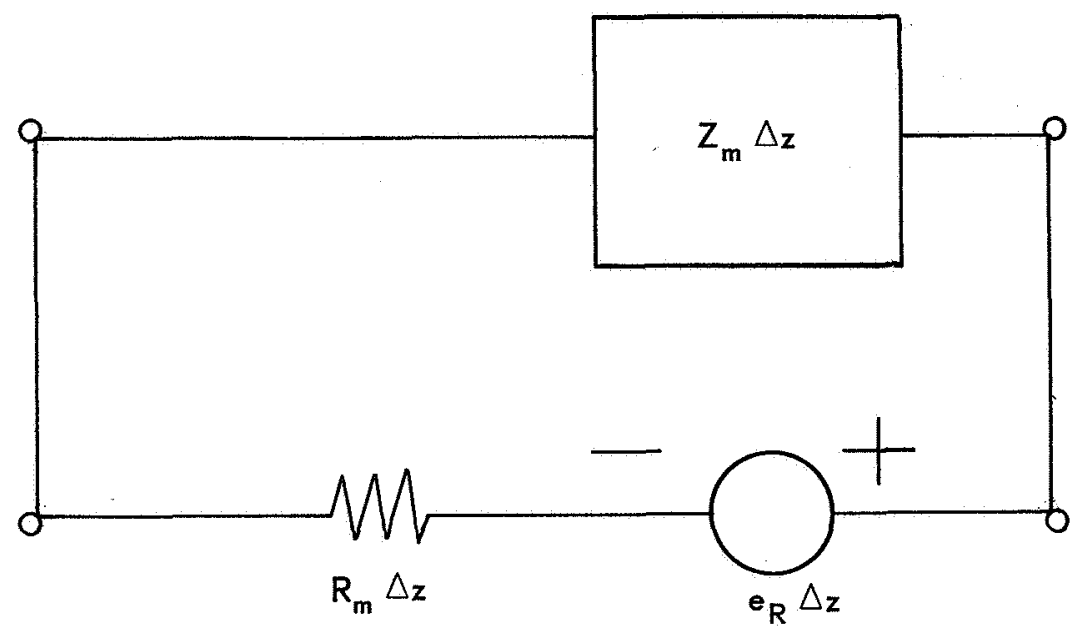

Figure 3(b). Circuit for Calculating Resistance Noise Power at Temperature $T_{m}$ Delivered to $Z_{m} \Delta z$.

The one-sided spectral-density of $e_{R} \Delta z$ is (Davenport and Root, (1958)) $4 \mathrm{kT}_{\mathrm{m}} \mathrm{R}_{\mathrm{m}} \Delta \mathrm{z}$, which implies that $\mathrm{S}_{\mathrm{e}_{\mathrm{R}}}(\omega)=4 \mathrm{kT} \mathrm{T}_{\mathrm{m}} \mathrm{R}_{\mathrm{m}} / \Delta \mathrm{z}$. Thus, (30) becomes

$$
\mathrm{S}_{\mathrm{m}}(\omega)=\frac{4 \mathrm{kT}}{\Delta \mathbf{z}} \cdot \frac{\omega \rho \alpha_{\mathrm{I}_{\mathrm{m}}}}{|1+a|^{2}}
$$

This is the spectral density of a source at temperature $T_{m}$ lying within the interval $(z, z+\Delta z)$. Such a source is statistically independent of all sources lying within any other intervals, no matter how fine we make such intervals. Thus, taking the limit $\Delta z \rightarrow 0$ in (31) we obtain for the expected value of $e_{m}\left(z_{1}\right) \cdot e_{m}^{*}\left(z_{2}\right):$

$$
\mathrm{E}\left[\mathrm{e}_{\mathrm{m}}\left(\mathrm{z}_{1}\right) \cdot \mathrm{e}_{\mathrm{m}}^{*}\left(\mathrm{z}_{2}\right)\right]=4 \mathrm{kT} \mathrm{m}_{\mathrm{m}} \frac{\omega \rho a_{\mathrm{I}_{\mathrm{m}}}}{|1+\alpha|^{2}} \delta\left(\mathrm{z}_{1}-\mathrm{z}_{2}\right),
$$

where $\delta\left(z_{1}-z_{2}\right)$ is the Dirac delta function. 
$\mathrm{kT}_{\mathrm{m}}$ is the low-frequency (or high-temperature) limit of the quantummechanical expression

$$
\frac{\hbar \omega}{\exp \left(\frac{\hbar \omega}{\mathrm{kT}_{\mathrm{m}}}\right)-1}
$$

which means that our final result for the expected value of $e_{m}\left(z_{1}\right) \cdot e_{m}^{*}\left(z_{2}\right)$ is

$$
E\left[e_{m}\left(z_{1}\right) \cdot e_{m}^{*}\left(z_{2}\right)\right]=\frac{4 \hbar \omega}{\exp \left(\frac{\hbar \omega}{k T_{m}}\right)-1} \cdot \frac{\rho \omega \alpha_{I_{m}}}{|1+\alpha|^{2}} \delta\left(z_{1}-z_{2}\right)
$$

This is the important relation between $\alpha_{I_{\mathrm{m}}}$ and the noise source at temperature $T_{m}$. Note that the frequency dependent coefficient of the delta function in (33) is always positive, regardless of the sign of $T_{m}$, because, as was shown above, $T_{m}$ and $\alpha_{I_{m}}$ take the same sign. Another important feature is that (33) has a peak at the resonant frequency of the $\mathrm{m}^{\text {th }}$ energy-level pair and then falls off to essentially zero outside the $2 / \tau$ bandwidth centered at the resonant frequency.

If the $i^{\text {th }}$ and $j^{\text {th }}$ energy levels together comprise the $m^{\text {th }}$ energy-level pair and if the no. density of ions occupying this pair of energy levels in $\mathrm{N}_{\mathrm{m}}$ then

$$
\begin{gathered}
n_{i}+n_{j}=N_{m} \\
n_{i}-n_{j} \exp \left(\frac{h \omega_{m}}{k T_{m}}\right)=0
\end{gathered}
$$


imply that

$$
n_{i}-n_{j}=N_{m}\left[\frac{\exp \left(\frac{\hbar \omega_{m}}{k T_{m}}\right)-1}{\exp \left(\frac{\hbar \omega_{m}}{k T_{m}}\right)+1}\right]
$$

where $\hbar \omega_{m}=E_{j}-E_{i}$

Upon comparing the imaginary part in (17) with (33) and (35), we see that (33) is proportional to

$$
N_{m}\left[\frac{\exp \left(\frac{\hbar \omega_{m}}{k T_{m}}\right)-1}{\exp \left(\frac{\hbar \omega_{m}}{k T_{m}}\right)+1}\right] \frac{4 \hbar \omega}{\exp \left(\frac{\hbar \omega}{k T_{m}}\right)-1}
$$

As $T_{m}$ approaches infinity this function approaches $2 N_{m} \hbar \omega$; it approaches zero as $T_{m} \rightarrow O_{+}$, and, finally, it approaches $4 N_{m} \hbar \omega$ as $T_{m} \rightarrow O_{-}$(complete population inversion). Each of these results is consistent with the fact that thermal noise of this type originates with ions occupying the higher energy level of a given pair. Such spontaneous emission noise is discussed with reference to electromagnetic masers in Siegman (1964).

Each generator associated with an impedance block in Figure 1(b) (see Figure 2) satisfies (33) with the appropriate temperature and $\alpha_{I}$. In addition such generators are statistically independent of each other, even though they lie within the same interval. Because of the series connection of impedance blocks 
and noise sources and because of the independence of these sources, it follows that the total noise voltage source, $e \Delta z$, is just the sum of each of the individual noise sources within the small interval and we have

$$
E\left[e\left(z_{1}\right) \cdot e^{*}\left(z_{2}\right)\right]=\sum_{m=1}^{P} E\left[e_{m}\left(z_{1}\right) \cdot e_{m}^{*}\left(z_{2}\right)\right],
$$

where $P$, again, stands for the total number of impedance blocks (which is equal to the total number of energy-level pairs in the paramagnetic spin system).

If we insert $e \Delta z$ in series with the series impedance in Figure 1(a) we can derive an inhomogeneous system of equations satisfied by the fluctuating field. Kirchoff's voltage and current laws give, respectively,

$$
\begin{aligned}
& \mathrm{V}(\mathrm{z}+\Delta \mathbf{z})=\mathrm{I}(\mathrm{z}+\Delta \mathbf{z}) \frac{\mathrm{j} \omega \rho \Delta z}{1+\alpha}+\mathrm{e} \Delta \mathbf{z}+\mathrm{V}(\mathbf{z}) \\
& \mathrm{I}(\mathbf{z}+\Delta \mathbf{z})=\mathrm{I}(\mathbf{z})+\mathrm{j} \frac{\omega}{\mu} \Delta \mathbf{z} \mathrm{V}(\mathbf{z}) .
\end{aligned}
$$

After rearrangement, division by $\Delta z$ and passage to the limit $\Delta z \rightarrow 0$, we get

$$
\begin{aligned}
& \frac{\mathrm{dV}}{\mathrm{d} z}=\mathbf{I} \frac{j \omega \rho}{1+\alpha}+\mathbf{e}(\mathbf{z}) \\
& \frac{\mathrm{dI}}{\mathrm{dz}}=\mathbf{j} \frac{\omega}{\mu} \mathrm{V}(\mathbf{z}) .
\end{aligned}
$$

Equation (38) differs from (22) only in the explicit inclusion of the composite noise source voltage-gradient $e(z)$. 
Finally, let us say a few words about the spectral density of the random sources associated with the complete vector system (21). We will consider the special case in which $a_{x x}=a_{y y}=a_{1}, a_{x y}=-a_{y x}=a_{2}$, because these conditions yield circularly polarized acoustic waves and have been the subject of some study (Muller and Tucker, 1967).

The space rate-of-change of time-average power along the transmissionline is given by

$$
\frac{\mathrm{dP}}{\mathrm{d} \mathbf{z}}=\left[\mathrm{I}_{\mathbf{x}}^{*}, \mathrm{I}_{\mathbf{y}}^{*}\right] \cdot j \omega \rho \frac{\left[\mathrm{A}^{-1}-\left(\mathrm{A}^{-1}\right)^{+}\right]}{2}\left[\begin{array}{l}
\mathrm{I}_{\mathbf{x}} \\
\mathrm{I}_{\mathbf{y}}
\end{array}\right],
$$

where $A$ is the matrix appearing in (21), $A^{-1}$ is its inverse, and the superscript dagger denotes conjugate-transpose.

By the assumed direction of current flow (right-to-left), the right-hand side of (39) must be non-negative for any $I_{x}, I_{y}$ if the transmission-line is dissipative (absorptive). A straight-forward, though tedious, calculation shows that $j \omega \rho\left[A^{-1}-\left(A^{-1}\right)^{+}\right]$is indeed a positive, semi-definite matrix for a normal (non-inverted) population.

Because we have a vector system we must associate two random funetions $e_{x}(z), e_{y}(z)$ with the dissipation matrix $j w \rho\left[A^{-1}-\left(A^{-1}\right)^{+}\right]$just as we associated a single source with the impedance $j \omega \rho / 1+a$. Corresponding to (33) 
we have the spectral density matrix

$$
\begin{array}{r}
E\left[\begin{array}{cc}
e_{x}\left(z_{1}\right) e_{x}^{*}\left(z_{2}\right) & e_{x}\left(z_{1}\right) e_{y}^{*}\left(z_{2}\right) \\
e_{x}^{*}\left(z_{1}\right) e_{y}\left(z_{2}\right) & e_{y}\left(z_{1}\right) e_{y}^{*}\left(z_{2}\right)
\end{array}\right]=\frac{4 \hbar \omega}{\exp \left(\frac{\hbar \omega}{k T}\right)-1} \\
\cdot j \omega p \frac{\left[A^{-1}-\left(A^{-1}\right)^{+}\right]}{2} \delta\left(z_{1}-z_{2}\right),
\end{array}
$$

which is positive semi-definite.

The inhomogeneous equations corresponding to (21) are

$$
\begin{aligned}
& \frac{d}{d z}\left[\begin{array}{l}
V_{x} \\
V_{y}
\end{array}\right]=j \omega \rho A^{-1}\left[\begin{array}{l}
I_{x} \\
I_{y}
\end{array}\right]+\left[\begin{array}{l}
e_{x} \\
e_{y}
\end{array}\right] \\
& \frac{d}{d z}\left[\begin{array}{l}
I_{x} \\
I_{y}
\end{array}\right]=j \frac{\omega}{\mu}\left[\begin{array}{l}
V_{x} \\
V_{y}
\end{array}\right] .
\end{aligned}
$$

\section{CALCULATION OF OUTPUT NOISE SPECTRAL DENSITIES}

As applications of the preceding developments we shall calculate the "voltage" and "current" (actually, strain and velocity) spectral densities for various configurations. The first configuration will be simply an infinite slab of unit crosssection, and we shall determine the spatial correlation function as well as spectral density. The other configurations will both be finite systems with a signal source at one end and the other end either free or terminated in the approximate characteristic impedance of the acoustic medium. In either of the 
latter two cases we will calculate the output spectral densities. The solutions, of course, are determined as boundary value problems.

Correlation and Spectral Density of Noise in an Infinite Region

We start with (38) expressed in vector-matrix form

$$
\frac{d \bar{V}}{d z}=\bar{M} \bar{V}+\bar{e}
$$

where

$$
\overline{\mathrm{V}}=\left[\begin{array}{l}
\mathrm{V} \\
\mathrm{I}
\end{array}\right], \quad \mathrm{M}=\left[\begin{array}{cc}
0 & \mathrm{j} \frac{\omega \rho}{1+\alpha} \\
\mathrm{j} \frac{\omega}{\mu} & 0
\end{array}\right], \quad \overline{\mathrm{e}}=\left[\begin{array}{l}
\mathrm{e} \\
0
\end{array}\right] .
$$

The solution of (42) is written in terms of a $2 \times 2$ Green's matrix, G(z- $\left.z^{\prime}\right)$ :

$$
\bar{V}(z)=\int_{-\infty}^{\infty} G\left(z-z^{\prime}\right) \bar{e}\left(z^{\prime}\right) d z^{\prime} .
$$

where $g\left(z-z^{\prime}\right)$ satisfies the matrix differential equation

$$
\frac{d G}{d z}-M G=\delta\left(z-z^{\prime}\right) U
$$

$\mathrm{U}$ is the $2 \times 2$ identity matrix. 
In component form (44) is

$$
\begin{aligned}
& V(z)=\int_{-\infty}^{\infty} g_{11}\left(z-z^{\prime}\right) e\left(z^{\prime}\right) d z^{\prime} \\
& I(z)=\int_{-\infty}^{\infty} g_{21}\left(z-z^{\prime}\right) e\left(z^{\prime}\right) d z^{\prime},
\end{aligned}
$$

$g_{11}$ and $g_{21}$. being elements of $G$.

After some manipulations and use of (33) and (36) we obtain for the spatial correlation functions of $V(z)$ and $I(z)$ :

$\mathbf{R}_{\mathrm{V}}(\mathrm{r}) \equiv \mathrm{E}\left[\mathrm{v}\left(\mathrm{z}_{1}\right) \mathrm{v}^{*}\left(\mathrm{z}_{2}\right)\right]=\left(\int_{-\infty}^{\infty} \mathrm{g}_{11}(\mathrm{r}+\zeta) \mathrm{g}_{11}^{*}(\zeta) \mathrm{d} \zeta\right)$

$$
\sum_{m=1}^{P} \frac{4 \hbar \omega}{\exp \left(\frac{\hbar \omega}{k T_{m}}\right)-1} \frac{\rho \omega \alpha_{I_{m}}}{|1+a|^{2}}
$$

$\mathbf{R}_{\mathrm{I}}(\mathrm{r}) \equiv \mathrm{E}\left[\mathrm{I}\left(\mathrm{z}_{1}\right) \mathrm{I}^{*}\left(\mathrm{z}_{2}\right)\right]=\left(\int_{-\infty}^{\infty} \mathrm{g}_{21}(\mathrm{r}+\zeta) \mathrm{g}_{21}^{*}(\zeta) \mathrm{d} \zeta\right)$

$$
\sum_{m=1}^{P} \frac{4 \hbar \omega}{\exp \left(\frac{\hbar \omega}{k T_{m}}\right)-1} \frac{\rho \omega \alpha_{I_{m}}}{|1+a|^{2}},(47)
$$


where $r=z_{1}-z_{2}$. These results show that the random processes $V(z), I(z)$ are stationary in space in the sense that their spatial correlations depend only on the difference $r=z_{1}-z_{2}$ and not an $z_{1}, z_{2}$, individually.

From here on we will assume that all energy-level pairs are at the same temperature so that the summation appearing in (47) is replaced by the single term

$$
\frac{4 \hbar \omega}{\exp \left(\frac{\hbar \omega}{\mathrm{kT}}\right)-1} \frac{\rho \omega \alpha_{I}}{|1+\alpha|^{2}}=S(\omega)
$$

Because the $\mathrm{V}$ and $\mathrm{I}$ processes are stationary it is convenient to introduce the wave-number representations, $\mathrm{H}_{\mathrm{V}}(\mathrm{k}), \mathrm{H}_{\mathrm{I}}(\mathrm{k})$, in order to calculate the correlation functions:

$$
\begin{aligned}
& H_{V}(k)=\int_{-\infty}^{\infty} R_{V}(r) e^{-j k r} d r \\
& H_{I}(k)=\int_{-\infty}^{\infty} R_{I}(r) e^{-j k r} d r .
\end{aligned}
$$

Upon substituting (47) (which are really convolution integrals in space) into (48) we obtain

$$
\begin{aligned}
& H_{V}(k)=S(\omega) G_{11}(k) G_{11}^{*}(k) \\
& H_{I}(k)=S(\omega) G_{21}(k) G_{21}^{*}(k)
\end{aligned}
$$


where $G_{11}(k)$ and $G_{21}(k)$ are spatial Fourier transforms, respectively, of $g_{11}(z), g_{21}(z)$ (the wave-number representation, (48), is, of course, the spatial Fourier transform).

The calculation of $G_{11}(k), G_{21}(k)$ proceeds most simply by taking the (spatial) Fourier transform of (45):

$$
(j k U-M) G(k)=e^{-j k z^{\prime}} U
$$

$G(k)$ is then given by

$$
\mathrm{G}(\mathrm{k})=\mathrm{e}^{-\mathrm{jkz} \mathrm{z}^{\prime}}(\mathrm{jkU} \mathrm{U}-\mathrm{M})^{-1}
$$

which, after substituting the matrix $M$, from (43), and identifying the 11 and 21 elements of the result, yields

$$
\begin{aligned}
& G_{11}(k)=\frac{j k e^{-j k z^{\prime}}}{-k^{2}+2\left(\frac{\rho}{\mu}\right) \frac{1}{1+\alpha}} \\
& G_{21}(k)=\frac{j \frac{\omega}{\mu} e^{-j k z^{\prime}}}{-k^{2}+\omega^{2}\left(\frac{\rho}{\mu}\right) \frac{1}{1+a}} .
\end{aligned}
$$


Upon substituting (52) into (49) and then taking the inverse transform of (48), we get

$$
\begin{aligned}
& \mathbf{R}_{\mathrm{V}}(\mathbf{r})=\frac{\mathrm{S}(\omega)}{2 \pi} \int_{-\infty}^{\infty} \frac{\mathbf{k}^{2} \mathrm{e}^{\mathrm{j} \mathbf{k} \mathbf{r}} \mathrm{dk}}{\left[\omega^{2}\left(\frac{\rho}{\mu}\right) \frac{1}{1+\alpha}-\mathrm{k}^{2}\right]\left[\omega^{2}\left(\frac{\rho}{\mu}\right) \frac{1}{1+\alpha^{*}}-\mathrm{k}^{2}\right]} \\
& \mathbf{R}_{\mathrm{I}}(\mathrm{r})=\frac{\mathrm{S}(\omega)}{2 \pi} \int_{-\infty}^{\infty} \frac{\left(\frac{\omega}{\mu}\right)^{2} \mathrm{e}^{\mathrm{j} \mathbf{k} \mathbf{r} \mathrm{dk}}}{\left[\omega^{2}\left(\frac{\rho}{\mu}\right) \frac{1}{1+\alpha}-\mathrm{k}^{2}\right]\left[\omega^{2}\left(\frac{\rho}{\mu}\right) \frac{1}{1+\alpha^{*}}-\mathrm{k}^{2}\right]} .
\end{aligned}
$$

We use residue theory (Churchill, 1960) to evaluate these integrals. The poles of the integrands are located at $a+j b, a-j b,-a-j b,-a+j b$ in the complex k-plane, where

$$
\begin{aligned}
& a=\omega\left[\frac{\left(\frac{\rho}{\mu}\right)^{2}}{|1+\alpha|^{2}}\right]^{1 / 4} \cos \left[\frac{1}{2} \tan ^{-1}\left(\frac{a_{I}}{1+\alpha_{R}}\right)\right] \\
& b=\omega\left[\frac{\left(\frac{\rho}{\mu}\right)^{2}}{|1+\alpha|^{2}}\right]^{1 / 4} \sin \left[\frac{1}{2} \tan ^{-1}\left(\frac{\alpha_{I}}{1+\alpha_{R}}\right)\right] .
\end{aligned}
$$

Note that the poles are symmetrically distributed about the real and imaginary axes.

We close the contour with a large semi-circle lying in the upper half-plane for $\mathrm{r}>0$ and lying in the lower half-plane for $\mathrm{r}^{\prime}<0$. This assures that contributions 
to the overall integral are negligible as the radii of the semi-circles go to infinity. The calculation of the residues is quite straight-forward with the result that

$$
\begin{aligned}
& R_{V}(r)=\frac{S(\omega)}{4 a b} e^{-b}|r|(a \cos a r-b \sin a|r|) \\
& R_{I}(r)=\frac{S(\omega)}{4 a b}\left(\frac{\omega}{\mu}\right)^{2} e^{-b}|r|\left(\frac{a \cos a r+b \sin a|r|}{a^{2}+b^{2}}\right) .
\end{aligned}
$$

The correlation functions are even in $\mathrm{r}$. We introduce the terms correlation length and correlation wavelength for $1 / \mathrm{b}$ and $2 \pi / \mathrm{a}$, respectively. Due to the presence of an imaginary part in $a$, we see that the farther apart in space two noise samples are measured (in the frequency domain) the less correlated they are.

Upon letting $r=0$ in (55) we obtain the spectral densities $S_{V}(\omega), S_{I}(\omega)$ of the $\mathrm{V}$ and I processes, respectively. Thus, substitution of (54) into (55) gives us

$$
\begin{aligned}
& \mathrm{S}_{\mathrm{v}}(\omega)=\frac{2 \hbar \omega}{\exp \left(\frac{\hbar \omega}{\mathrm{kT}}\right)-1} \cdot \frac{(\mu \rho)^{1 / 2}}{|1+a|^{1 / 2}} \cos \left[\frac{1}{2} \tan ^{-1}\left(\frac{\alpha_{\mathrm{I}}}{1+\alpha_{\mathrm{R}}}\right)\right] \\
& \mathrm{S}_{\mathrm{I}}(\omega)=\frac{2 \hbar \omega}{\exp \left(\frac{\hbar \omega}{\mathrm{kT}}\right)-1} \cdot \frac{|1+\alpha|^{1 / 2}}{(\mu \rho)^{1 / 2}} \cos \left[\frac{1}{2} \tan ^{-1}\left(\frac{\alpha_{\mathrm{I}}}{1+\alpha_{\mathrm{Rx}}}\right)\right]
\end{aligned}
$$


These results admit the following interesting interpretation. From Figure 1(a) the "inductance" per-unit-length is seen to be $\rho / 1+\alpha$ and the "capacitance" per-unit-length is $-1 / \mu$. Hence, the magnitude of the wave impedance is

$$
\left(\frac{\frac{\rho}{|1+a|}}{\frac{1}{\mu}}\right)^{1 / 2}
$$

Therefore, upon dividing $S_{V}(\omega)$ by (57) or multiplying $S_{I}(\omega)$ by the same function we get the spoctral-densities of the power associated with the "voltage" and "current" processes, respectively. The power spectral-densities are equal and their sum is

$$
S_{p}(\omega)=\frac{4 \hbar \omega}{\exp \left(\frac{\hbar \omega}{k T}\right)-1} \cos \left[\frac{1}{2} \tan ^{-1}\left(\frac{\alpha_{I}}{1+\alpha_{R}}\right)\right]
$$

If the spins are brought into thermal equilibrium with the acoustic medium at temperature $\mathrm{T}$, and then if the medium is made nondispersive by setting $\alpha(\omega)=0$, we get the phonon thermal equilibrium result

$$
S_{p}(\omega)=\frac{4 \hbar \omega}{\exp \left(\frac{\hbar \omega}{k T}\right)-1}
$$


Our results are general in that they contain this result as a special case. The corresponding result for electromagnetic radiation in 3-dimensional space is given in Landau and Lifshitz (1960).

\section{Signal Source and Free Termination}

Consider a signal source connected at $z=0$ and the end $z=L$ left free. If we take the actual (acoustic) source to be an imposed strain then the electrical analogue is a voltage source. The free surface at $z=L$ appears electrically to be a short-circuit because a free surface cannot support a shear-stress. Since stress is given by

$$
\mu \frac{\partial u}{\partial z}+\frac{\partial M}{\partial z}=\mu(1+a) \frac{\partial u}{\partial z}=(1+a) v
$$

we see that $V(L)=0$, hence, the short-circuit termination.

Our concern is with the calculation of the spectral-density of the current (velocity) at $z=\mathrm{L}$ due to the signal and noise. We take the signal and noise to be uncorrelated. We start with (38) and the boundary conditions

$$
\begin{aligned}
& V(0)=E_{s}(\omega) \\
& V(L)=0,
\end{aligned}
$$

where $\mathrm{E}_{\mathrm{s}}(\omega)$ is the Fourier transform of the "voltage" signal source connected at $z=0$. 
The output current will be a superposition of the currents arising from $E_{s}$ and the noise source $e(z)$, each acting alone. We label the voltage and current arising from $E_{s}, V_{s}$ and $I_{s}$, while that resulting from $e(z), V_{n}$ and $I_{n}, V_{s}$ and $I_{s}$ satisfy (38) with $e(z)$ set equal to zero and the boundary conditions (59). $V_{n}, I_{n}$ satisfy (38) and the boundary conditions $V_{n}(0)=V_{n}(L)=0$. Then $V=V_{n}+V_{s}$ and $I=I_{n}+I_{s}$ satisfy (38) and (59).

It is a simple matter to show that $V_{s}$ and $I_{s}$ are given by

$$
\begin{aligned}
& V_{s}(z)=E_{s}(\omega) \frac{\sin k(L-z)}{\operatorname{sinkL}} \\
& I_{s}(z)=j\left(\frac{E_{s}}{Z_{0}}\right) \frac{\cos k(L-z)}{\operatorname{sinkL}},
\end{aligned}
$$

where

$$
\mathrm{k}=\omega\left(\frac{\rho}{\mu(1+\alpha)}\right)^{1 / 2} \quad \text { and } \quad \mathrm{z}_{0}=\left(\frac{\mu \rho}{1+\alpha}\right)^{1 / 2}
$$

We next postulate a noise solution of the form

$$
\begin{aligned}
& V_{n}(z)=\int_{0}^{L} g_{V}\left(z, z^{\prime}\right) e\left(z^{\prime}\right) d z^{\prime} \\
& I_{n}(z)=\int_{0}^{L} g_{I}\left(z, z^{\prime}\right) e\left(z^{\prime}\right) d z^{\prime}
\end{aligned}
$$


with the voltage and current Green's functions satisfying

$$
\begin{aligned}
\frac{\mathrm{dg}_{\mathrm{I}}}{\mathrm{dz}} & =\mathrm{j} \frac{\omega}{\mu} \mathrm{g}_{\mathrm{V}} \\
\frac{\mathrm{dg} \mathrm{V}}{\mathrm{d} z} & =j \frac{\omega \rho}{1+\alpha} g_{I}+\delta\left(z-z^{\prime}\right) \\
\frac{\left.\mathrm{dg}_{\mathrm{I}}\right|_{\left(0, z^{\prime}\right)}}{\mathrm{dz}} & =\left.\frac{\mathrm{dg}_{\mathrm{I}}}{\mathrm{dz}}\right|_{\left(L, z^{\prime}\right)}=0 .
\end{aligned}
$$

The boundary conditions on $d_{g} / d z$ are a result of the conditions on $V_{n}$. Implicit in (62) are conditions of continuity of $g_{I}$ at $z=z^{\prime}$ and discontinuity of amount $j \omega / \mu$ in the derivative of $g_{I}$ at $z=z^{\prime}$.

The result of applying these conditions to (62) is that

$$
\begin{array}{rlrl}
g_{I}\left(z, z^{\prime}\right) & =j \frac{\omega \cos k\left(z^{\prime}-L\right) \cos k z}{k \sin k L}, & \\
z^{\prime}<z^{\prime} \\
& =j \frac{\omega}{\mu} \frac{\cos k z^{\prime} \cos k(z-L)}{k \sin k L}, & z>z^{\prime} \\
g_{V}\left(z, z^{\prime}\right) & =\frac{-\sin k z \cos k\left(z^{\prime}-L\right)}{\sin k L}, & z z^{\prime} \\
& =\frac{-\sin k(z-L) \cos k z^{\prime}}{\sin k L}, & z>z^{\prime} .
\end{array}
$$


The noise solutions thus become

$$
\begin{aligned}
& V_{n}(z)=-\int_{0}^{z} \frac{\sin k(z-L) \cos k z^{\prime}}{\sin k L} e\left(z^{\prime}\right) d z^{\prime} \\
& -\int_{z}^{L} \frac{\sin k z \cos k\left(z^{\prime}-L\right)}{\sin k L} e\left(z^{\prime}\right) d z^{\prime} \\
& I_{n}(z)=j \frac{\omega}{\mu}\left[\int_{0}^{z} \frac{\cos k z^{\prime} \cos k(z-L)}{k \sin k L} e\left(z^{\prime}\right) d z^{\prime}\right. \\
& \left.+\int_{z}^{L} \frac{\cos k\left(z^{\prime}-L\right) \cos k z}{k \sin k L} e\left(z^{\prime}\right) d z^{\prime}\right]
\end{aligned}
$$

The total output current (velocity) at $z=L$ is obtained by adding $I_{s}(L)$ and $I_{n}(L):$

$$
I(L)=\frac{j}{Z_{0} \sin k L}\left[E_{s}+\int_{0}^{L} \cos k z^{\prime} e\left(z^{\prime}\right) d z^{\prime}\right] .
$$

The spectral density $S_{I}(\omega)$ of $I(L)$ is obtained easily by using the fact that $E_{s}$ and $e(z)$ are uncorrelated (i.e., $E\left[E_{s} e\right]=0$ ) and also using (33) and (36). Hence, upon multiplying (65) by its complex conjugate and taking the expected 
value we obtain

$$
S_{I}(\omega)=\frac{1}{\left|Z_{0}\right|^{2}|\sin k L|^{2}}\left[S_{s}(\omega)+\left(\int_{0}^{L}|\cos k z|^{2} d z\right)\right.
$$

$$
\left.\sum_{m=1}^{P} \frac{4 \hbar \omega}{\exp \left(\frac{\hbar \omega}{k T_{m}}\right)-1} \frac{\rho \omega \alpha_{I_{m}}}{|1+a|^{2}}\right]
$$

where $S_{s}$ is the signal spectral-density.

Since the integrand appearing in (66) is non-negative it follows that increasing $\mathrm{L}$, the length of the acoustic medium, results in a larger noise contribution to the output current (velocity). This is to be expected because each elementary noise generator within the structure contributes noise independently to the output. Therefore, the longer the structure the more contributing noise sources present.

In accordance with (26), the expected mean-square value of the output current is given by the integral (66) overall positive frequencies. If the signal applied at $z=0$ is $E_{s} \cos \omega_{s} t$ then $S_{s}(\omega)=\pi E_{s}^{2} \delta\left(\omega-\omega_{s}\right)$ and

$$
E\left[\overline{\left.\mathrm{i}_{0}^{2}\right]}=\frac{\mathrm{E}_{\mathrm{s}}^{2}}{2\left|\mathrm{Z}_{0}\right|^{2}\left|\sin \mathrm{k}\left(\omega_{s}\right) \mathrm{L}\right|^{2}}+\frac{1}{2 \pi} \int_{0}^{\infty} \mathrm{N}(\omega) \mathrm{d} \omega\right.
$$

where $N(\omega)$ is the second term in (66). By choosing $L$ appropriately it is possible to make the denominator of the first term in (67) small (if $k\left(\omega_{s}\right)$ were 
real, we could make the denominator vanish) and therefore amplify the signal, without amplifying all frequency components of the noise, simply by resonating the structure at the signal frequency.

\section{$\underline{\text { Signal Source and Matched Termination }}$}

In this case we will assume that $|\alpha|<<\mid$. Under this condition $Z_{0}$, the characteristic impedance of the spin-phonon system, is approximately $(\mu \rho)^{1 / 2}$, which we take to be the terminating impedance.

The boundary conditions are

$$
\begin{aligned}
& \mathrm{V}(0)=\mathrm{E}_{\mathrm{s}} \\
& \mathrm{V}(\mathrm{L})=-\mathrm{I}(\mathrm{L}) \cdot(\mu \rho)^{1 / 2},
\end{aligned}
$$

which are also the boundary conditions satisfied by $V_{s}$ and $I_{s^{*}}$ The boundary conditions satisfied by the noise are

$$
\begin{aligned}
& V_{n}(0)=0 \\
& V_{n}(L)=-I_{n}(L) \cdot(\mu \rho)^{1 / 2} .
\end{aligned}
$$

The Green's function $g_{I}$ satisfies the boundary conditions

$$
\left.\frac{\mathrm{dg}_{\mathrm{I}}}{\mathrm{dz}}\right|_{\left(0, z^{\prime}\right)}=0,\left.\quad \frac{\mathrm{dg}_{\mathrm{I}}}{\mathrm{dz}}\right|_{\left(L, z^{\prime}\right)}=-\left.\mathrm{j} \omega\left(\frac{\rho}{\mu}\right)^{1 / 2} \mathrm{~g}_{\mathrm{I}}\right|_{\left(L^{\prime}, z^{\prime}\right)},
$$


and the differential equations in (62). The solutions for $g_{I}$ and $g_{V}$ are

$$
\begin{aligned}
& g_{I}\left(z, z^{\prime}\right)=\frac{-\cos k z^{\prime} e^{-j k z}}{k} \cdot \frac{\omega}{\mu}, \quad z^{\prime}<z \\
& =\frac{\mathrm{e}^{-\mathrm{jkz} \mathbf{z}^{\prime} \cos \mathrm{kz}}}{\mathrm{k}} \cdot \frac{\omega}{\mu}, \quad \mathrm{z}^{\prime}>\mathrm{z} \\
& g_{V}\left(z, z^{\prime}\right)=\cos k z^{\prime} e^{-j k z}, \quad z^{\prime}<z \\
& =j e^{-j k z^{\prime}} \sin k z, \quad z^{\prime}>z, \\
& \mathrm{k}=\omega\left(\frac{\rho}{\mu(1+\alpha)}\right)^{1 / 2} .
\end{aligned}
$$

The total output voltage and current are given by

$$
\begin{aligned}
& V(L)=V_{s}(L)+V_{n}(L)=E_{s} e^{-j k^{\prime} L}+e^{-j k L} \int_{0}^{L} \cos k z^{\prime} e\left(z^{\prime}\right) d z^{\prime} \\
& I(L)=I_{s}(L)+I_{n}(L)=-\frac{E_{s}}{(\mu \rho)^{1 / 2}} e^{-j k L}-\frac{e^{-j k L}}{(\mu \rho)^{1 / 2}} \int_{0}^{L} \cos k z^{\prime} e\left(z^{\prime}\right) d z^{\prime} .
\end{aligned}
$$

In deriving (72) we have used the approximate value of $Z_{0}$, stated earlier, and have set reflections equal to zero. 
The spectral densities of (72) are

$S_{v}(\omega)=\exp \left(2 k_{I} L\right)\left[S_{S}(\omega)+\left(\int_{0}^{L}|\cos k z|^{2} d z\right) \sum_{m=1}^{P} \frac{4 \hbar \omega}{\exp \left(\frac{\hbar \omega}{k T_{m}}\right)-1} \cdot \frac{\rho \omega a_{I_{m}}}{|1+\alpha|^{2}}\right]$ $=\exp \left(2 k_{I} L\right) S(\omega)+\left[\frac{\exp \left(4 k_{I} L\right)-1}{4 k_{I}}+\frac{\exp \left(2 k_{I} L\right) \sin 2 k_{R} L}{4 k_{R}}\right]$.

$\sum_{m=1}^{P} \frac{4 \hbar \omega}{\exp \left(\frac{\hbar \omega}{k T_{m}}\right)-1} \frac{\rho \omega \alpha_{I_{m}}}{|1+\alpha|^{2}}$

$\mathbf{S}_{\mathrm{I}}(\omega)=\frac{\mathbf{S}_{\mathbf{v}}(\omega)}{\mu \rho}$.

$\mathrm{k}_{\mathrm{I}}, \mathrm{k}_{\mathrm{R}}$ in (73) (the imaginary and real parts, respectively, of $\mathrm{k}$ ) are given in terms of $a$ by $-b$, a, respectively, of (54). As a check on (73) we note that if each $T_{m}=T>0$ then in the limit $L \rightarrow \infty, \alpha \rightarrow 0$, in that order, we derive the results (56) for an infinite medium.

For a signal spectrum concentrated at $\omega_{\mathrm{s}}$

$$
E\left[\overline{\left.v_{0}^{2}\right]}=\frac{E_{s}^{2}}{2} \exp \left(2 k_{I}\left(\omega_{s}\right) L\right)+\frac{1}{2 \pi} \int_{0}^{\infty} N(\omega) d \omega\right.
$$

where $N(\omega)$ is the second term in (73). 
If $k_{I}\left(\omega_{s}\right)$ is positive due to a population inversion of the energy-level pair whose frequency separation is $\omega_{\mathrm{s}}$, then we have amplification at the signal frequency (the phonon maser). A population inversion implies that for some $\mathrm{m}$ in (73), $\alpha_{I_{m}}$ and $T_{m}$ are both negative. $N(\omega)$, however, remains positive, as it should. It should also be noted in (73) that $N(\omega)$ vanishes at those frequencies for which $a_{I_{m}}$ vanishes. In our discussion of $\alpha_{I_{m}}$ we pointed out that it was non-zero essentially only in a bandwidth $2 / \tau_{l_{m}}$ wide, centered at $\omega \ell_{m^{*}}$ Hence, this is the band of frequencies which contribute to $N(\omega)$. 


\section{REFERENCES}

1. Bleaney, B. and Stevens, K. W. H., "Repts. Progr. in Phys.," 16, 108 (1953).

2. Bowers, K. D. and Owen, J., "Repts. Progr. in Phys.," 18, 304 (1955).

3. Churchill, R. V., "Complex Variables and Applications," New York: McGraw-Hill, 1960.

4. Cooper, G. R. and McGillem, C. D., "Methods of Signal and System Analysis," New York: Holt, Rinehart and Winston, 1967.

5. Davenport, Jr., W. B. and Root, W. L., "An Introduction to the Theory of Random Signals and Noise," New York: McGraw-Hill, 1958.

6. deGroot, S. R. and Mazur, P., "Non-Equilibrium Thermodynamics," New York: Interscience, 1962.

7. Haus, H. A., "Thermal Noise in Dissipative Media," J. App. Phys., 32 , 493 (1961).

8. Jacobsen, E. H., "Sources of Sound in Piezoelectric Crystals," J. Acoust. Soc. Am., 32, 949 (1960).

9. Landau, L. D. and Lifshitz, E. M., "Statistical Physics," Reading: AddisonWesley, 1958 .

10. Landau, L. D. and Lifshitz, E. M., "Electrodynamics of Continuous Media," Reading: Addison-Wesley, 1960.

11. Morse, P. M. and Feshbach, H., "Methods of Theoretical Physics," Part I, New York: McGraw-Hill, 1953. 
12. Muller, E. R. and Tucker, J. W., "Acoustic-Wave Rotation by Spin-Phonon Interaction," Con. J. Phys., $\underline{45}, 2443$ (1967).

13. Sabbagh, H. A., "Maser Spin Dynamics," GSFC (NASA) Document X-523-66-448, Sept. 1966.

14. Seavey, Jr., M. H., "Phonon Generation by Magnetic Films," Proc. IEEE, $\underline{53}, 1387$ (1965).

15. Siegman, A. E., "Microwave Solid-State Masers," New York: McGrawHill, 1964.

16. Slichter, C. P., "Principles of Magnetic Resonance," New York: Harper and Row, 1963.

17. Tucker, E. B., "Interaction of Phonons with Iron-Group Ions," Proc. IEEE, $\underline{53}, 1547(1965)$.

18. Twiss, R. Q., "Nyquist's and Thevenin's Theorems Generalized for Nonreciprical Linear Networks," J. App. Phys., 26, 599 (1955).

19. Van Kranendonk, J. and Lee, Y. Y., "Theory of the Spin-Lattice Interaction in Paramagnetic Salts of Iron-Group Ions," Can. J. Phys., 44, 1613 (1966).

20. Van Wormhaudt, M. and Haus, H. A., "Thermal Noise in Linear, Lossy, Electromagnetic Media," J. App. Phys., 33, 2572 (1962). 